\title{
Le Quinquatrus, una festa di Minerva
}

\author{
Tiziano Cinaglia \\ Doctor por la Università degli Studi di Perugia \\ tiziano.cinaglia@virgilio.it
}

Recibido: 27 de octubre de 2015

Aceptado: 28 de noviembre de 2015

\begin{abstract}
RIASSUNTO
La letteratura moderna ha generalmente interpretato la festività delle Quinquatrus come una celebrazione propria di Marte, in base all'attività saliare certificata da alcuni feriali antichi per il 19 marzo. In realtà, da numerosi ed evidenti indizi, le Quinquatrus devono considerarsi come una festa di esclusiva pertinenza di Minerva, oltretutto già a partire dall'epoca arcaica: in primo luogo, il dies natalis della dea è ricordato da varie fonti per questa data; ma rivestono una importanza decisiva, ai fini dell'individuazione della titolarità della festa, soprattutto l'analisi etimologico-lessicale del sostantivo Quinquatrus e le relazioni mitologico-calendariali sottese a questa festività. La concomitante presenza dei rituali dei Salii durante le celebrazioni dedicate a Minerva, pertanto, altro non è che una chiara testimonianza della stretta connessione tra il culto di Marte e quello della dea, nel primo mese dell'anno arcaico.
\end{abstract}

Parole chiave: Quinquatrus. Minerva. Dies natalis. Salii. Calendario lunare. Anna Perenna.

\section{Quinquatrus, a festival of Minerva}

\begin{abstract}
Modern scholars have generally interpreted the festivity of Quinquatrus as a celebration pertinent to Mars, as some ancient feriali certify the activity of Salii priests for 19 March. Actually, by numerous and evident clues, the Quinquatrus must be considered as a festival dedicated exclusively to Minerva, moreover, already since the archaic era: first of all, the dies natalis of the goddess is mentioned by different sources on this date; but, for the identification of the ownership of this festival, it is of decisive importance especially the lexical and etymological analysis of the noun Quinquatrus and the calendarial and mythological relationships that the festivity denotes. Therefore, we have to interpretate the concomitant presence of rituals of Salii during the celebrations dedicated to Minerva only as a clear testimony of the close connection between the cult of Mars and that of the goddess, in the first month of the archaic year.
\end{abstract}

Keywords: Quinquatrus. Minerva. Dies natalis. Salii. Lunar calendar. Anna Perenna.

Sommario: 1. Dies natalis. 2. Salii faciunt in Comitio saltu. 3. Sia Feriae Martis che feriae Minervae? 4. 'Il quinto giorno scuro'. 5. Da 'quinto giorno scuro' a Quinquatrus. 6. Minerva e Anna Perenna. 7. Conclusioni. 


\title{
1. Dies natalis ${ }^{1}$
}

\author{
Una dies media est et fiunt sacra Minervae \\ nominaque a iunctis quinque diebus habent. \\ Sanguine prima vacat nec fas concurrere ferro; \\ causa, quod est illa nata Minerva die. ${ }^{2}$
}

È questo l'incipit dell'oltremodo noto brano ovidiano dedicato alle Quinquatrus: dopo aver dato la sua spiegazione sull'origine del nome della festa -errata, come vedremo- il poeta dei Fasti definisce esplicitamente questo giorno come il dies natalis della dea; ed anche l'antiquaria romana, sebbene alquanto confusamente, era altrettanto consapevole che quella data costituisse il 'natale' della dea: infatti, sempre al 19 marzo, sono ricordate, secondo due diverse e discordanti tradizioni, le dedicationes del tempio del Celio in un primo caso, ed in un secondo del santuario aventinese. ${ }^{3} \mathrm{La}$ letteratura moderna si è abbastanza equamente suddivisa a favore dell'una o dell'altra teoria: secondo una prima corrente, il 19 marzo rappresenterebbe il giorno della dedicatio del santuario di Minerva sull'Aventino, mentre la data del 19 giugno, che presenta la particolarità di essere simmetrica al 19 marzo, ad un intervallo di tre mesi, corrisponderebbe invece alla constitutio dello stesso santuario: ${ }^{4}$ in questo modo, di conseguenza, verrebbe spiegato il suo doppio anniversario. Secondo una differente teoria, invece, le due date si potrebbero giustificare con un restauro e quindi una nuova dedicatio del santuario aventinese, ${ }^{5}$ effettuata ad opera di Augusto appunto il giorno 19 giugno; ${ }^{6}$ Gilbert, Warde Fowler e Brelich, invece, consideravano il 19 mar-

1 L'espressione dies natalis, generalmente traducibile con il significato di 'compleanno' (vd. TLL, IX, 1.1, 122 ss), poteva però essere impiegata anche relativamente alla dedica di una struttura templare e, quindi, alla 'nascita' della divinità che ne era titolare (Ov., Fast., III, 837-838: Parva licet videas Captae delubra Minervae / quae dea natali coepit habere suo; TLL, IX, 1.1, 125s.; Varr., in Non., 209 Lindsay, L. 2: Si hodie noenum venis, cras quidem si veneris meridie die natali Fortis Fortunae).

2 Ov., Fast., III, 809-812.

3 Le due contrapposte tradizioni sono capeggiate, per così dire, l'una da Ovidio e l'altra da Verrio Flacco: è infatti il poeta dei Fasti (III, 837-838), sempre all'interno del medesimo brano sulle Quinquatrus, a specificare come la data del 19 marzo rappresentasse la dedicatio del tempio ceriolense; concordemente con questa affermazione, nel VI libro Ovidio assegnerà la consacrazione del tempio dell'Aventino al 19 giugno, a 3 mesi esatti di distanza dal tempio più antico del Celio (VI, 725-728: Iam sex et totidem luces de mense supersunt, / Huic unum numero tu tamen adde diem: / Sol abit a Geminis et Cancri signa rubescunt; / Coepit Aventina Pallas in arce coli). A supporto di questa tradizione vi sono anche le testimonianze dei Fasti Antiates maiores, degli Esquilini e degli Amiternini (Degrassi, Inscr. It., XIII 2, rispettivamente alle pagine 6-7, 88, 187). Nei Fasti Prenestini, al contrario, Verrio Flacco sostiene che il 19 marzo rappresentasse la dedica del tempio aventinese (vd. nota 18), seguito poi in questa ipotesi anche dal suo epitomatore Festo (vd. nota 42).

4 Mommsen in CIL I' $\mathrm{I}^{2}$, 312s. e 320; Degrassi, Inscr. It., XIII 2, 472; JoRdAN - Huelsen 1970-71, I,3, 159, n. 24; Bömer 1957-58, II, 200; Platner - Ashby 1965, 342; CANCiani 1984, 1075; SChÜrmanN 1985, 9; SCHILLING 1992, 160.

5 Aust 1889, 19; Wissowa 1902, 203; Ross Taylor 1923, 75, n. 52; Platner-Ashby, 342; Girard 1981, 206; ArCella 1991, 45. Questa ipotesi, d'altronde, non può avere alcun credito, in quanto smentita dal fatto che i Fasti Preanestini sono stati redatti posteriormente ai Fasti Esquilini. Girard, d'altro canto, in tempi più recenti ha rivisto la propria posizione in merito, mettendo in dubbio che il santuario aventinese potesse avere il 19 marzo come dies natalis e pertanto concordando con la successiva ipotesi avanzata da Torelli (GIRARD 1989, 164).

6 August., RGDA, 19, 2: aedes Minervae ... in Aventino ... feci. 
zo come il dies natalis di entrambi i templi, sia dell'aventinese che del ceriolense. ${ }^{7}$ $\mathrm{Ma}$ in realtà, se esaminiamo più attentamente la notizia tramandataci da Festo relativamente al tempio dell' Aventino, notiamo che essa è riportata dall'autore "con una nota di distanza dall'opinione dell'antiquario prenestino", come giustamente rimarca Torelli: ${ }^{8}$ infatti il verbo usato, existimant, non denota una totale certezza dell'affermazione, forse perché l'altra sua fonte a disposizione in merito, probabilmente Varrone, riportava una tradizione differente. ${ }^{9}$ Dobbiamo pertanto convenire con Torelli che questa moderna interpretazione, che ipotizza due date diverse per la dedicatio e la constitutio, rappresenti in realtà una forzatura; al contrario è più logico ritenere la spiegazione verriana come una notizia creata $a d$ hoc, a giustificare un rapporto, quello tra Quinquatrus e artifices, ormai consolidato in età tardo-repubblicana, ma le cui origini risultavano però incerte. ${ }^{10}$ In conclusione, ritengo che si debba ammettere con Ovidio, seguendo il più antico feriale romano, che il 19 marzo fosse il dies natalis di Minerva Capta e il 19 giugno una successiva dedica del tempio di Minerva in Aventino; ipotesi, questa, che negli ultimi decenni è divenuta ormai quella più generalmente accettata. ${ }^{11}$

Quindi, in definitiva, tradizione letteraria e antiquaria romana, sebbene in forme diverse, concordavano nell'attribuire il dies natalis della dea al 19 marzo, il giorno in cui si celebravano annualmente le Quinquatrus; o meglio, giorno in cui, in età tardo-repubblicana, iniziava il periodo di cinque giorni di festeggiamenti che terminava il 23 dello stesso mese con la celebrazione del Tubilustrium. ${ }^{12}$ Per il momento, pertanto, dobbiamo rilevare la coincidenza tra il 'compleanno' di Minerva e la

7 Gilbert 1883-1890, II, 233s., n. 2; Warde Fowler 1899, 59; Brelich 1949, 110, n. 65.

8 Torelli 1984, 55.

9 Le incertezze della tradizione si evidenziano anche e soprattutto nei dubbi ovidiani sull'origine del culto di Minerva Capta (Fast., III, 839-847).

10 Come abbiamo già sottolineato, la dedicatio del tempio aventinese del 19 giugno ha una stretta relazione, in quanto simmetricamente collocata a tre mesi esatti di distanza, con le Quinquatrus di marzo; ma le Quinquatrus minusculae, anch'esse dedicate a Minerva, cadevano in realtà il 13 giugno, alle idi del mese, e non in corrispondenza con la dedica del tempio dell'Aventino. D'altronde, la tradizione letteraria romana dava della duplicazione del culto delle Quinquatrus una spiegazione miti-storica, legata alla vicenda dei tibicines, al loro auto-esilio ed alla loro successiva reintegrazione nel corpo sacerdotale romano, una esegesi che non contribuiva di certo a fare chiarezza sul rapporto tra Minerva e le Quinquatrus.

11 Dello stesso avviso sono De SANCTIS (1953, IV.2.1, 146, n. 73, 147), Torelli (1984, 54s.), ZiOLKOWSKI (1992, 109, 113s.), Coarelli (1996, 255 e 1998, 212), Donati - Stefanetti (2006, 38) e Scheid (2008, 85), mentre Richardson riconosce la data del 19 marzo come dies natalis del tempio del Celio, ma rimane dubbioso sulla dedicatio di quello aventinese (RICHARDSON 1992, 255). La duplicazione del culto di Minerva a giugno porterà anche ad una duplicazione della stessa festa delle Quinquatrus, e sarà probabilmente questo sdoppiamento della principale festività della dea a determinare le incertezze degli autori antichi sul dies natalis dei due templi, dal momento che il culto principale della dea in età tardo-repubblicana, molto probabilmente, era ormai divenuto quello dell'Aventino e non più quello del Celio, sia per la monumentalità del santuario (FUR, tav. XXIII, 22 b), che invece doveva certamente mancare al parvum delubrum del Celio, sia per la centralità che dovette assumere, in seguito alla concessione a scribae ed histriones del 207 a.C. di celebrarvi la propria festa (Liv., XXVII, 37, 1-10; Fest., 446L), nei festeggiamenti e nei rituali connessi per le varie corporazioni artigianali cittadine.

12 Sappiamo da un brano di Livio che, per lo meno già dalla prima metà del II sec. a.C., le Quinquatrus duravano più di un singolo giorno (Liv., XLIV, 20, 1: His intra triduum simul cum legatis Alexandrinis profectis legati ex Macedonia quinquatribus ultimis adeo expectati venerunt, ut, nisi vesper esset, extemplo senatum vocaturi consules fuerint): si tratta infatti di un evento del 168 a.C., preludio alla notissima battaglia 
festività delle Quinquatrus, un indizio del nesso tra questa celebrazione ed il culto della dea: infatti, evemeristicamente, la festa principale di una divinità, o la dedicatio del suo santuario più importante, era generalmente considerata anche la sua data di nascita. Ma l'antiquaria romana era altresì concorde nell'attribuire a Minerva anche tutti i festeggiamenti, tutti gli atti di devozione, pratiche e rituali che i cittadini romani compivano in questa giornata; le testimonianze a riguardo sono innumerevoli, a partire già dall'età medio-repubblicana, ${ }^{13} \mathrm{e}$ ci descrivono le Quinquatrus come una celebrazione gioiosa e allegra, dal carattere ludico, oserei dire 'leggero', nella quale il ruolo centrale era giocato dalle corporazioni artigianali che, sotto la tutela di Minerva, celebravano il loro artificum dies. ${ }^{14}$ Ma questi seppur numerosi documenti del patronato della dea sulle Quinquatrus non possono costituire una prova definitiva della pertinenza di questa festività a Minerva sin dai suoi exordia; al contrario, ritengo non sia possibile dimostrare che le celebrazioni di questa giornata risalissero sin al periodo arcaico già sotto l'egida, è proprio il caso di dirlo, della dea: infatti, come abbiamo precedentemente ricordato, la fonte più antica che menziona questa festa in riferimento a Minerva è quella di Plauto, pertanto non anteriore alla fine del III sec. a.C.; ed inoltre, la prima menzione di questa celebrazione in relazione ad un evento storicamente accertato è, scherzi del destino, praticamente contemporanea alla precedente, in quanto databile per l'appunto alla fine del III secolo, all'anno 210 a.C. ${ }^{15}$

\section{Salii faciunt in Comitio saltu ${ }^{16}$}

Ciò non ostante, ossia prescindendo dalle numerose testimonianze sulle celebrazioni di questa festività, ritengo che la correlazione Minerva-Quinquatrus si possa leggere tra le pieghe della storia, evidenziata da vari indizi; dello stesso avviso, però, non è la vulgata moderna, comunemente concorde nel sostenere la recenziorità della presenza della dea relativamente alle celebrazioni del 19 marzo. Ma questa affermazione non deriva dall'esame della festa stessa; d'altronde, sarebbe impossibile provare il contra-

di Pidna contro il re Perseo, nel quale alcuni legati romani, di ritorno dalla Macedonia, giungono a Roma negli 'ultimi giorni delle Quinquatrus'.

13 La prima attestazione delle Quinquatrus è di Plauto (Mil., 691-693: da, mi vir, Calendis meam qui matrem munerem; / da qui faciam condimenta; da quod dem Quinquatribus / praecantrici, coniectrici, hariolae atque haruspicae ...).

14 È la definizione impiegata per questa data da Verrio Flacco nei Fasti Praenestini (vd. nota 18). La testimonianza di maggior rilievo per la conoscenza delle attività connesse alle Quinquatrus è ovviamente il brano dei Fasti ovidiani (III, 809-848); ma le fonti che apportano ulteriori dettagli, quantunque minimi, contribuendo a delineare un quadro preciso di questa festa, sono decisamente numerose ed in questa sede mi limito semplicemente ad elencarle, senza però entrare nel dettaglio di una loro analisi, ché sarebbe estremamente lunga e non pertinente al tema in esame (Caes., BG, VI, 17, 1-2; Front., Als., 3, 10; Juv., Sat., X, 114-117; Hier., in Eph., VI, 4; Hor., Ep., II, 2, 197-198; Lact., Inst., I, 18, 23; Macr., Sat., I, 12, 6; Nov., Atell., 95; Ov., Am., III, 2, 52; Plin., NH, XXXV, 143; Porph., II, 2, 197; Sym., Ep., 85, 3; Suet., Aug., LXXI, 5; Ner., 34, 2; Symm., Ep., V, 85; Tac., Ann., XIV, 12; Tert., Idol., 10; Varr., Re.Rust., III, 2, 18; Men., frg. 440).

15 Liv. XXVI, 27, 1: Interrupit hos sermones nocte quae pridie Quinquatrus fuit pluribus simul locis circa forum incendium ortum. Ci troviamo nel periodo della guerra annibalica; l'evento in questione è l'incendio che distrusse parzialmente il foro e nel quale bruciarono le tabernae argentariae.

16 Vd. nota 18. 
rio, dal momento che la data del 19 marzo in epoca storica era letteralmente 'monopolizzata' da Minerva; in effetti, gli studiosi moderni hanno sempre dichiarato, con rare eccezioni, che la dea avesse 'usurpato' le Quinquatrus a Marte in età tardo-repubblicana, acquisendo immediatamente una preminenza assoluta sino ad estromettere del tutto lo stesso Marte da queste celebrazioni, partendo da un unico presupposto, da un unico dato, desumibile dall'analisi degli antichi feriali conservatici: ${ }^{17}$ questa festa sarebbe da attribuirsi esclusivamente a Marte, per quanto concerne il periodo arcaico, semplicemente sulla scorta di una nota dei Fasti Praenestini di Verrio Flacco, i quali riportano per il 19 marzo dei rituali, delle danze, che venivano celebrate nel Comizio ed erano connesse all'attività saliare; questa teoria sarebbe per di più corroborata da un secondo dato, un'ulteriore nota, questa volta presente nei Fasti Vaticani, che rubricava questo giorno come feriae Martis. ${ }^{18}$ Secondo questa interpretazione, dunque, Minerva si sarebbe 'appropriata' della festività delle Quinquatrus soltanto in epoca tardo-repubblicana in qualità di dea delle arti, a motivo della accresciuta importanza di artisti ed artigiani nella vita cittadina romana: a questo proposito, come ulteriore certificazione di questa affermazione, si fa generalmente riferimento ad un evento del 207 a.C., anno nel quale, per merito di Livio Andronico e del carmen espiatorio da lui composto, viene concesso agli scribae ed agli histriones di poter consistere ac dona ponere nel santuario della Minerva aventinese; ${ }^{19} \mathrm{~d}$ 'altronde, quasi coeva è anche la 'data di nascita' convenzionale della letteratura romana, ancora una volta protagonista Livio Andronico, con la prima commedia in latino rappresentata a Roma nel 240 a.C. Il prestigio e la centralità di artisti e artigiani sul finire del III sec. a.C., dunque, sarebbe stata la causa del passaggio di consegne tra Marte e Minerva, una divinità

17 Sono numerosi gli studiosi che sostengono questa ipotesi, a partire da WARDE FowLER (1899, 57), Wissowa (1899, 1554; ID. 1902, 203) e Hentschel (1963, 1154ss.), per continuare poi con De SanCTIS (1953, IV.2.1, 148), Hild (1969a, 802), Frazer (1973, 144), con la Storchi Marino (1979, 344 e 348), DurYMoyaers $(1981,228)$, Scullard $(1981,93)$, con Canciani $(1984,1074$ s.), la Porte $(1985,85$ e 122$)$, con York (1986, 101s.), SAbBAtucci (1988, 133), BAYet (1992, 100), Hinds (1992, 101), la InVERnizZi (1994, 41), RüPKe (1995, 79s. e 499), COARELli (1998, 212), CARANDini (2003, 559ss.), con Donati - SteFANETTI $(2006,36)$, La Bua $(2010,47)$, Moret $(2012,53$ ss.) ed infine con la Granino Cecere $(2014,108)$. Tutti gli studiosi concordano nell'affermare che questa 'interferenza' di Minerva si sarebbe verificata soltanto in età tardo-repubblicana, dopo la presa di Falerii del 241 a.C., o piuttosto verso la fine del III sec., in seguito al citato episodio di Livio Andronico.

18 I Fasti Praenestini riportano la dicitura $[Q V I N] Q \cdot N$, e successivamente un lungo commento: recte tamen alii putarunt dictum ab eo quod hic dies est post diem u idus quod $\cdot$ in Latio $\cdot$ post idus dies simili ratione declinarentur / artificum dies quod Minervae aedis $\cdot$ in Aventino $\cdot$ eo $\cdot$ die $\cdot$ est dedicata / Salii faciunt $\cdot$ in comitio $\cdot$ saltu adstantibus pontificibus $\cdot$ et $\cdot$ trib $\cdot$ celer (DEGRASSI, Inscr. It. XIII 2, 122s.); i Fasti Vaticani, invece, riportano la dicitura $Q V I N Q \cdot N P$ e quindi la nota feriae Mar(ti) (DEGRAssI, Inscr. It. XIII 2, $173=$ CIL I $\left.{ }^{2}, 1,242\right)$. Questi ultimi, rinvenuti a Roma nel 1779, sono databili al regno di Tiberio sulla base di dati interni al testo; i Fasti Praenestini, invece, recuperati a più riprese tra il 1770 ed il 1864 nella cittadina laziale di Palestrina, sono databili alla tarda età augustea (vd. RüPKE 1995, 79s. e 114ss.).

19 Liv., XXVII, 37, 7; Fest., 446L. Si tratta dell'espiazione di un prodigium, o meglio di vari prodigia, che avevano sconvolto Roma e che, per ordine dei pontefici, furono placati per mezzo di un inno composto da Livio Andronico e cantato da 27 vergini. La notizia liviana del prodigium, e della successiva espiazione (Liv., XXVII, 37, 1-10: ... Decrevere item pontifices, ut virgines ter novena per urbem euntes carmen canerent. Id cum in Iovis Statoris aede discederent conditum ab Livio poeta carmen, tacta de caelo aedis in Aventino Iunonis Reginae ...), ci viene completata da Festo, che la collega al tempio dell'Aventino (446L: Itaque cum Livius Andronicus bello Punico secundo scripsisset carmen ... publice adtributa est ei in Aventino aedis Minervae, in qua liceret scribis histrionibusque consistere ac dona ponere). 
ormai totalmente ellenizzata, 'doppione' della greca Atena, dea dell'intelletto e delle arti.

Ma in questa per quanto circostanziata analisi, in realtà, non si è mai tenuto conto di un elemento fondamentale, di un dato facilmente individuabile anche agli occhi di un 'non addetto ai lavori': mi sto riferendo all'istituzione, o meglio alle origini miti-storiche, delle Quinquatrus minusculae, celebrate annualmente il 13 giugno e calco di quelle principali di marzo, ed alle logiche deduzioni che questa duplicazione comporta nel discorso più generale sulle Quinquatrus. Le testimonianze letterarie a riguardo sono numerose e la descrizione dell'evento, quantunque con dettagli diversi e con un maggior o minor grado di approfondimento, è essenzialmente uniforme ed univoca: ${ }^{20}$ si tratta della nota vicenda dell'esilio volontario dei tibicines a Tivoli, come forma di protesta nei confronti del senato che aveva drasticamente ridotto i loro privilegi; l'episodio, datato al 311 a.C., ${ }^{21}$ ha un 'lieto fine', in quanto i suonatori delle tibiae $^{22}$ verranno successivamente reintegrati nel corpo sacerdotale cittadino con le proprie originarie prerogative, ma solo dopo esser stati ricondotti a Roma, ubriachi e mascherati da donne, e lasciati all'alba nel Foro. Le caratteristiche di questa celebrazione sono molto particolari, con evidenti connotati di licenziosità e ribaltamento dell'ordine precostituito, ${ }^{23}$ quello che è però fondamentale porre in evidenza, in realtà, è che già da queste scarne informazioni possiamo ricavare un dato incontrovertibile: le Quinquatrus minusculae nascono come festività connessa al culto di Minerva. Ma da questa seppur banale considerazione, possiamo però giungere ad una logica quanto sostanziale conclusione: infatti, la creazione delle Quinquatrus minuscualae implica, credo sia evidente, che il nesso Minerva-Quinquatrus già esistesse in precedenza, e che altresì fosse ben radicato nella communis opinio romana, per lo meno già a partire dal IV sec. a.C., se non addirittura in un'epoca ancora anteriore. ${ }^{24}$ In

20 Cens., De die nat., XII, 2; De Vir. Ill., 34, 1; Fest., 134L; Liv., IX, 30, 5-10; Ov., Fast., VI, 651-710; Plut., Quaest.Rom., 55; Quint., Inst., V, 11, 9; Varr., Ling.Lat., VI, 17.

21 In quest'anno, infatti, i censori, tra i quali doveva esserci anche Appio Claudio Cieco, proibirono ai flautisti di consumare il pasto sacro nel tempio di Giove Capitolino e ridussero il numero dei tibicines a 10 unità (anche se probabilmente questa limitazione era già presente nelle Dodici Tavole; Cic., Leg., 2, 59); come segno di protesta, in conseguenza alla limitazione delle loro prerogative, i flautisti attuarono questo sciopero ante litteram, abbandonando Roma e recandosi a Tivoli.

22 L'uso della tibia, giunta a Roma tramite il mondo etrusco (Liv., VII, 2, 4), era fondamentale per lo svolgimento di qualsiasi cerimonia religiosa, in quanto il suo suono era in grado di placare gli dei (Cens., 12; Arn., VII, 32); secondo la tradizione, l'istituzione dei tibicines risalirebbe a Numa (Plut., Numa, 17, 3) o piuttosto a Tarquinio Prisco (Strab., V, 2, 2). Su tibia e tibicines vd. VetTer 1936, 808ss.; ReinACH 1969a, 300ss.; VENDRIES 2004, 398 e 405ss.

23 In questa sede non ci è possibile esaminare approfonditamente le peculiari caratteristiche di questa vicenda, a metà tra mito e storia, né tanto meno le complesse implicazioni di carattere cultuale che doveva comportare questa 'mascherata' per le vie della città (per le fonti vd. nota 20). Ad oggi, l'ipotesi più convincente è quella che spiega questa particolare celebrazione con dei rituali legati alla 'stagione dell'aurora e di quanto la prepara e la condiziona' (GRANINO CECERE 2003, 80), connessi inoltre strettamente al solstizio d'estate (DumÉZIL 1973, 174ss.; Humm 2005, 441ss.); è però di notevole interesse la recente proposta della Buchet, che vede nel travestitismo dei tibicines e nel loro esilio a Tivoli un legame con il culto di Ercole (BUCHET 2010, 183ss. - per una bibliografia aggiornata e per una sintesi delle varie ipotesi sull'argomento vd. ibidem, 174ss.).

24 Le fonti pongono la nascita delle Quinquatrus minusculae alla fine del IV sec., ma vi sono vari indizi per ritenere erronea questa collocazione cronologica: infatti, come si può facilmente evincere dai testi sopra citati, al rientro dei tibicines a Roma viene loro concesso nuovamente di banchettare nel tempio di Giove; non 
altre parole, dobbiamo decisamente convenire che le Quinquatrus maiores fossero già di pertinenza della dea verosimilmente già agli inizi del periodo repubblicano; conseguentemente, ritengo che non sia in alcun modo ormai più accoglibile l'ipotesi di una associazione tardiva di Minerva a questa festa, solo sulla base di un aumentato prestigio delle arti e dell'ingenium alla fine del III sec. a.C. Non è pertanto necessario continuare ad ipotizzare una sovrapposizione, ed infine una definitiva sostituzione, del culto di Minerva a quello di Marte in epoca tardo-repubblicana; tutt'al più, con la Storchi Marino, potremmo ammettere l'ipotesi che il culto della dea avesse soppiantato quello di Marte già in una fase alta, addirittura nel V sec. a.C., divenendo le Quinquatrus una festività di esclusiva pertinenza di Minerva sin dagli inizi del periodo repubblicano. ${ }^{25}$

Però, in merito a quest'ultimo tema, credo sia necessario fare una ulteriore osservazione: che il giorno 19 marzo si celebrasse il culto di Marte ad opera dei Salii è indubitabile, come documentato dalle fonti antiquarie; ed il rituale saliare, d'altronde, era fortemente compenetrato a tutto il mese, a partire dal primo giorno di marzo, quando i Salii si recavano alla Curia sul Palatino per invocare Marte e prendere i dodici ancilia, coi quali si sarebbero poi recati alla Regia per assistere al solenne sacrificio offerto dal pontifex maximus; l'attività saliare poteva dirsi terminata il 24 marzo, giorno in cui il rex sacrorum dichiarava chiusa la serie dei giorni religiosi. ${ }^{26} \mathrm{Ma}$ la

vi è però cenno alcuno, in nessuna fonte, né a Minerva né alle eventuali sue connessioni con i flautisti (l'unica ad evidenziarlo è la Buchet 2010, 182s.); e la data stessa della festività, il 13 giugno, ossia le idi del mese, la collega piuttosto nuovamente al culto Giove (Fasti Venusini e Tuscolani - DegRAssI, Inscr. It. XIII 2, 58 e 103; Ov., Fast., VI, 649-650). Come ipotesi di lavoro, se da un lato la celebrazione presenta indubbi connotati di arcaicità, ritengo però che, d'altro canto, si possano più verosimilmente ritenere sia la denominazione di 'Quinquatrus minusculae' sia il patronato di Minerva su di essa come delle innovazioni posteriori (dello stesso avviso la STORCHI MARINO 1979, 349); e potremmo forse datare con buona probabilità il passaggio di consegne tra Giove e Minerva alla seconda metà del III sec. a.C. quando, in seguito all'evocatio del culto di Minerva da Falerii del 241 a.C. ed alla conseguente dedicatio di una nuova struttura templare in onore della dea, si verranno così a costituire a Roma un nuovo luogo ed un nuovo culto, in altre parole una 'nuova' Minerva, 'disponibile' ad accogliere rituali e celebrazioni di questa festività in quanto patrona dei musicisti, in vesti ormai totalmente ellenizzate (in questa sede vale la pena ricordare che il mito di Marsia e dell'invenzione del flauto da parte di Atena era ben noto a Roma già per lo meno dagli inizi del III secolo a.C., quando una statua del satiro campeggiava nei Comitium, dedicata probabilmente da C. Marcius Rutilus Censorinus, censore del 294 a.C. - Penny Small 1982, 68ss.; Torelli 1982, 98ss.; Coarelli 1985, 91ss.; Richardson 1992, 371).

25 Storchi Marino 1979, 344 e 348.

26 Sul tema delle celebrazioni, dei rituali e del mito dei Salii, tra gli innumerevoli contributi, sono da menzionare: Hild 1969b, 1014ss.; Martínez-Pinna 1980, 15ss.; Torelli 1984, 23ss. e 107ss.; Coarelli 1985, 303ss.; SabBatucci 1988, 93ss.; Guidobaldi 1992, 14s.; Borgna 1993, 28ss.; Bremmer 1993, 160ss.; MaIURi 2009, 149ss.; Moret 2012, 49ss.; Granino CeCERe 2014, 105ss. La compenetrazione tra i rituali saliari legati al culto di Marte e le celebrazioni riservate a Minerva deve probabilmente risalire alla fase arcaica della religione romana: siamo infatti a conoscenza dell'esistenza dei versus Minervii, una espressione che è traducibile come 'canti di Minerva', o 'inni di Minerva', preghiere che insieme agli axamenta facevano parte dei componimenti dei Carmina Saliaria (Paul. Fest., 3L: Axamenta dicebantur carmina Saliaria, quae a Saliis sacerdotibus componebantur, in universos homines composita. Nam in deos singulos versus ficti a nominibus eorum appellabantur, ut Ianuli, Iunonii, Minervii); ora, questi testi sono evidentemente di notevole antichità, in quanto fissati per iscritto nel IV secolo a.C. ma tramandati oralmente già a partire da una fase molto più alta, e risultavano ormai incomprensibili agli stessi Romani di epoca primo-imperiale (Quint., I, 6, 40-41), poiché composti in una lingua decisamente arcaica. Pertanto, se Minerva poteva essere 'cantata', celebrata, in un contesto di così alta arcaicità, all'interno del rituale saliare, è evidente che anche il suo stesso culto e la sua stessa presenza a Roma debbano considerarsi necessariamente altrettanto risalenti, quanto meno contemporanei, se 
presenza dei Salii in quanto tale, non è certamente un motivo sufficiente per estromettere Minerva, per giustificarne e quindi certificarne l'assenza dalle origini delle Quinquatrus; se così fosse, infatti, allo stesso modo dovremmo mettere in discussione anche la pertinenza dei Matronalia a Giunone Lucina, dal momento che il $1^{\circ}$ marzo è il giorno in cui prendono avvio le celebrazioni saliari: in effetti, la leggenda fissava proprio in questa giornata la caduta miracolosa dal cielo dell'ancile, ${ }^{27}$ ed inoltre $\mathrm{i}$ calendari indicano per questa data la nota feriae Martis, ${ }^{28}$ così come è riportato anche per il 19 marzo; infine, sempre al primo giorno del mese di marzo, era addirittura collocato anche il dies natalis del dio guerriero. ${ }^{29}$ Non vi è evidentemente bisogno di sottrarre a Giunone 1'originario patronato dei Matronalia per spiegare la contestuale attività saliare; d'altro canto, la contemporanea presenza di più celebrazioni in una stessa data non era infrequente nel feriale romano. ${ }^{30}$ Allo stesso modo neppure si rende necessario leggere, semplicisticamente, nella compresenza delle Quinquatrus e dei rituali saliari una sovrapposizione di diversi culti di epoca tarda; pertanto, dal confronto tra le due differenti ma allo stesso tempo analoghe situazioni, ritengo che non vi sia motivo alcuno per ammettere, come al contrario sostiene la maggior parte della letteratura moderna, che si sia verificato un tale passaggio di competenze tra Marte e Minerva per quanto riguarda le Quinquatrus.

Da ultimo, se torniamo nuovamente ad esaminare gli antichi fasti romani, credo che da questa analisi si possa desumere un ulteriore e fondamentale elemento per l'attribuzione della pertinenza di Minerva alle Quinquatrus, sin dalle sue origini: infatti, così come i due feriali precedentemente citati riportano per il 19 marzo delle feriae in onore di Marte, altri due calendari, però, menzionano esclusivamente la nostra dea

non precedenti, alla formazione ed alla formulazione di questi versus sacri (Bayet ipotizza che gli inni dedicati a Giunone e Minerva furono aggiunti in seguito alla dedicatio del tempio capitolino; BAYET 1992, 87). Come spunto di riflessione, vorrei infine aggiungere una ulteriore considerazione: la vulgata moderna ha spesso sostenuto l'estraneità di Minerva all'originario pantheon romano puntando il dito sull'assenza di un flamen dedicato alla dea (De Sanctis 1953, IV.2.1, 146; Jullian 1969, 1167; CANCiani 1984, 1074; Graf 2001, 131); ma se esaminiamo l'elenco dei 15 flamines (Fest., 145L; in realtà due dei quindici cognomina dei flamines minores non sono documentati), non si può non notare la contemporanea assenza di un flamen anche per Giano e per Giunone, assieme alla mancanza di un 'flamen minervalis'. Ora, se torniamo ad analizzare il passo di Festo sugli axamenta e lo esaminiamo sinotticamente con l'elenco dei flamines, credo si possa evidenziare una singolare complementarità tra i due testi, sinora non altrimenti rilevata: infatti, se da un lato non esistevano flamines per Giano Giunone e Minerva, dall'altro però troviamo citati dei versus saliari esclusivamente per queste tre divinità. Pertanto, si può forse ipotizzare che le differenti connotazioni dei due sacerdozi, flamonium e saliatus, li rendessero in un certo qual modo 'complementari' ma, al contempo, ne limitassero anche le loro sfere di competenza solo e soltanto ad alcune divinità; dunque, come Giano e Giunone, anche Minerva doveva avere un culto a Roma già in epoca arcaica, ma evidentemente strettamente collegato al rituale saliare, come è evidente dal suo inserimento nelle festività del ciclo di marzo, piuttosto che con le attività dei flamines.

27 Liv., I, 20, 2; Ov., Fast., III, 365-392; Plut., Numa, I, 13.

28 I Fasti Praenestini riportano la dicitura $K \cdot M A R T N P$, e quindi il commento: feriae $\cdot$ Marti $\cdot$ Iun [o]ni . Lucinae $\cdot$ Esquiliis quod $\cdot e o \cdot$ die $\cdot$ aedis $\cdot$ ei [dedica]ta $\cdot$ est $\cdot$ per $\cdot$ matronas quam voverat Albin [ia] ... vel . uxor $\cdot$ si $\cdot$ puerum ... que ipsa (DEGRASSI, Inscr. It., XIII 2, 122s.).

29 I Fasti Philocaliani riportano $N \cdot$ MARTIS (Degrassi, Inscr. It., XIII 2, 243; SalzMan 1990, 122-126).

$30 \mathrm{Nel}$ solo mese di marzo, ad esempio, si celebravano contestualmente i seguenti culti: Marte in campo e Giunone Lucina, nei Matronalia, il primo giorno del mese; le festività di Equirria e Mamuralia il 14; Anna Perenna e Giove, in quanto titolare delle idus, il 15; infine, i Liberalia, dedicate a Liber Pater, e l'Agonium di Marte, il 17. La compresenza di Minerva e Marte nella data del 19 marzo, di conseguenza, non costituisce in alcun modo un'eccezione nel feriale arcaico. 
come 'titolare' delle celebrazioni di questa giornata: nello specifico, stiamo parlando dei Fasti Farnesiani e dei Fasti Antiates Maiores. ${ }^{31}$ Ora, è noto che i Fasti Antiates Maiores, in quanto unico calendario documentatoci anteriore alla riforma di Giulio Cesare, vengano generalmente considerati come un 'residuo fossile' dell'originario feriale della comunità romana, come l'unico documento affidabile capace di restituirci una immagine fide digna della religione arcaica di Roma; ${ }^{32}$ la validità di questa testimonianza, pertanto, è notevolmente maggiore rispetto ai documenti ad essa cronologicamente posteriori e che collegano la festa al culto di Marte; cconseguentemente, il fatto che le Quinquatrus siano rubricate in questo contesto come proprie di Minerva, deve essere interpretato come prova certa dell'arcaicità del rapporto tra questa festività e la dea. Per fugare ulteriori dubbi, inoltre, è utile evidenziare anche un ultimo particolare: infatti, nei Fasti Antiates Maiores, soltanto il nome della festa è riportato con lettere capitali grandi, mentre il nome della dea è in realtà rubricato con caratteri più piccoli; si potrebbe quindi forse obiettare che la presenza di Minerva sia da considerarsi un'aggiunta posteriore. Ma a questo riguardo va però osservato che, in questo feriale, allo stesso modo vengono rubricate le feste di divinità quali, ad esempio, Carmenta, Quirino, Cerere, Mater Matuta, divinità la cui arcaicità non è certamente in discussione. Dobbiamo pertanto convenire che le Quinquatrus pertenessero a Minerva sin dal periodo arcaico, riconsiderando quindi le teorie che escludono a priori l'antichità del culto della dea a Roma e la sua presenza nel feriale antico; d'altronde, già alla metà del secolo scorso Brelich sosteneva che la festività delle Quinquatrus dovesse appartenere sin dalle origini sia a Marte che a Minerva, con celebrazioni rivolte singolarmente alle due divinità, proposta successivamente accolta da vari studiosi. ${ }^{33}$

31 I Fasti Antiates Maiores riportano la dicitura QVIN Minervae (Degrassi, Inscr. It., XIII 2, 7), mentre i Fasti Farnesiani riportano la dicitura QVIN Minerv[ae] (DegrassI, Inscr. It., XIII 2, 225); questi ultimi, di provenienza probabilmente urbana, sono genericamente databili al periodo primo-imperiale (RüPKE 1995, 76s.).

32 Coarelli 2010, 338. I Fasti Antiates Maiores, rinvenuti ad Anzio nel 1915, costituiscono l'unico documento anteriore alla riforma giuliana del calendario del 46 a.C.; sono databili tra l's a.C., ultimo anno in cui nel calendario vengono indicati i consoli, ed il 55 a.C.: infatti, l'omissione dei culti di Venus Victrix, Honos, Virtus e Felicitas, celebrati nel teatro di Pompeo, suggerisce di datare la redazione dei fasti anteriormente alla costruzione del teatro stesso. Secondo Rüpke il modello dei Fasti Antiates Maiores può risalire al massimo agli inizi del II sec. a.C., per la presenza delle consonanti doppie (Ann(ae) Perennae al 15 marzo, [Al]liens(is) $\operatorname{die}(s)$ al 18 luglio), probabilmente introdotte a Roma da Ennio (RüPKE 1995, 339ss.).

33 Brelich 1949, 110, n. 65. Dello stesso avviso sono anche LatTe (1960, 164 e 435), la Storchi Marino (1979, 346), Arcella (1991, 27), RadKe (1993, 140s.), Phillips $(2006,942)$ e LipKa (2009, 40s.). Anche Graf è concorde nel riconoscere l'antichità della presenza di Minerva nelle celebrazioni delle Quinquatrus: egli, infatti, ritiene che questa festa appartenga ad uno strato arcaico della religione romana e che debba essere stata introdotta dagli artigiani (ceramisti e scultori) greci, nello specifico euboici, attraverso Cuma (GRAF 2001, 138). Un'ipotesi, quest'ultima, sicuramente da respingere, come dimostreremo più avanti. 


\section{Sia Feriae Martis che feriae Minervae?}

Ma anche l'affermazione che le Quinquatrus fossero una festa di entrambe le divinità, per quanto abbia il merito di riconoscere l'antichità della presenza di Minerva in questa celebrazione, non è del tutto convincente; credo, infatti, che vi siano indizi sufficienti per sostenere che il dio guerriero non giocasse alcun ruolo nelle celebrazioni delle Quinquatrus e che questa, pertanto, fosse una festa esclusivamente di Minerva, sin dalle origini. ${ }^{34}$ La prima prova a favore di questa tesi si individua ad un esame delle varie festività del ciclo saliare, ponendo particolare attenzione alla loro denominazione e cercando così eventuali attinenze o differenze con il sostantivo Quinquatrus; ora, le altre date del culto dei Salii nel mese di marzo sono rispettivamente: il $1^{\circ}$ marzo, giorno dei Matronalia e dies natalis di Marte; il 9 marzo, per il quale sempre il calendario di Filocalo riporta la nota arma, ancilia movent $;^{35}$ la data del 14 marzo, giorno in cui si celebravano gli Equirria; ${ }^{36}$ ed infine il 23 marzo, ovvero il giorno del Tubilustrium. ${ }^{37}$ Quello che appare immediatamente evidente è che non tutte le date delle celebrazioni saliari avevano una specifica denominazione che le contraddistinguesse, dei nomi che le identificassero, come nel caso del $1^{\circ}$ e del 9 marzo; le altre due date rimanenti, ovvero le ultime due festività sopra elencate, sono caratterizzate da altrettanti nomi propri, Equirria e Tubilustrium. ${ }^{38}$ Di conseguenza, la festa delle Quinquatrus sembrerebbe poter essere accostata a queste ultime, in

34 Si badi bene, stiamo parlando esclusivamente del ruolo che la vulgata moderna attribuisce a Marte per le Quinquatrus, non del ruolo che il dio evidentemente aveva nelle celebrazioni che, sempre nella data del 19 marzo, erano però connesse ai rituali saliari.

35 Degrassi, Inscr. It., XIII 2, 243.

36 Gli Equirria, o Ecurria, erano una festa arcaicissima, risalente addirittura a Romolo (Paul. Fest., 81L), che consisteva in una corsa di cavalli nel Campo Marzio, il 27 febbraio ed il 14 marzo (Ov., Fast., II, 858860; Varr., Ling.Lat., VI, 13). Si trattava di una festa di sacralizzazione dei cavalli all'inizio della campagna militare, per propiziare la stagione guerriera, ma anche, in coppia con 1'altra festa degli Equirria del 27 febbraio ed inserita in un più ampio contesto di celebrazioni di fine anno, a favorire il passaggio dal vecchio al nuovo anno. Vd. sul tema LoicQ 1964, 491s.; SABBATUCCI 1988, 82 e 97s.; RADKe 1993, 130s.; CoARELli 1997, 61ss.; Bernstein 1999, 149ss.; Woodard 2002, 96ss.; Blaive 2003, 284ss.; Martínez-Pinna 2012, 162 ss.

37 Festività nota da varie fonti (Fasti Praenestini, Degrassi, Inscr. It. XIII 2, 123; Fest., 482L; Lyd., de Mens., IV, 60; Ov., Fast., III, 849-850; Varro, Ling.Lat., VI, 14). Il Tubilustrium, celebrato nell'atrium Sutorium, era il giorno adibito alla purificazione delle trombe liturgiche, le tubae, dedicate a Marte ed usate in varie occasioni, ma particolarmente in ambito funerario e bellico; gli attori principali di questa giornata erano i tubicines sacrorum, sacerdoti o piuttosto officianti consacrati, insieme ovviamente ai Salii, che in questa giornata riconducevano gli ancilia nella Regia. Il Tubilustrium di marzo, in onore di Marte (DEGRASSI, Inscr. It. XIII 2, 104 e 123), indice di una chiara relazione con la sfera bellica, era però in stretta relazione con l'altro Tubilustrium, celebrato il 23 maggio e dedicato invece a Vulcano (DEGRASSI, Inscr. It. XIII 2, 57 e 187): in questa seconda celebrazione lustrale, al momento della chiusura della più antica campagna militare, venivano offerte a Vulcano le armi nemiche, per essere consacrate e defunzionalizzate attraverso il fuoco (sul tema e sulle funzioni dei due Tubilustria vd. ReINACH 1969b, 526ss.; Storchi Marino 1979, 337ss.; ScUllard 1981, 94s.; Torelli 1984, 66ss. e 100s.; ID. 1990, 95ss.; SABBATUCCI 1988, 113ss. e 211s.; MAGDELAIN 1990, 275ss.; RADKe 1993, 141s.; RÜPKe 1995, 214ss.; CoARelli 1997, 66; LiOU-Gille 2000, 58; MarCATTILi 2011, 254ss.).

38 Ovviamente, nell'elenco delle festività dei Salii non si tiene conto, come precedentemente esposto, della festa dei Matronalia, che deve necessariamente essere considerata come una festa solo e soltanto di pertinenza di Giunone, non avendo l'attività saliare, certificata per questo giorno, alcuna diretta attinenza con questa celebrazione. 
quanto anch'essa identificata da un nome proprio; ma in realtà notiamo prontamente come vi sia una sostanziale differenza tra il termine Quinquatrus ed i nomi di Equirria e Tubilustrium: questi ultimi due, in effetti, hanno già intrinseco nel nome un riferimento esplicito ai rituali connessi con il culto di Marte e con il culto saliare: se da un lato gli Equirria prevedevano una corsa di cavalli nel Campo Marzio in onore di Marte, dall'altro il Tubilustrium consisteva nella purificazione delle trombe liturgiche, dai connotati militari oltre che sacrali. Nella parola quinquatrus, al contrario, non si può rilevare, né tanto meno soltanto scorgere, alcun rimando alle pratiche dei Salii o al culto di Marte; ${ }^{39}$ come vedremo successivamente, infatti, il nome deve essere letto con il significato di 'quinto giorno scuro', espressione nella quale non si può riconoscere alcuna particolare connessione con il rituale saliare. Credo, pertanto, non sia azzardato ipotizzare che la festività delle Quinquatrus, seppur inserita nel ciclo saliare, avesse un'origine diversa da quest'ultimo, come sembra trasparire da questa prima analisi; ma d'altro canto, se è molto probabile che l'etimologia e quindi l'origine delle Quinquatrus siano da ricercare altrove, in ogni caso il suo inserimento all'interno del ciclo saliare e nelle festività d'inizio anno non può ritenersi comunque casuale e privo di significato, bensì doveva corrispondere ad una stretta correlazione e corrispondenza tra queste celebrazioni e l'originario significato della festa delle Quinquatrus.

Ma se ampliamo questa comparazione di natura etimologico-lessicale a tutto il ciclo delle 45 feste fisse che caratterizzavano il calendario pre-giuliano, notiamo ancora più marcatamente la particolarità, direi l'unicità, di questo sostantivo, che immediatamente risalta nel panorama delle celebrazioni arcaiche; ${ }^{40}$ in effetti, la denominazione delle festività 'numane' generalmente derivava dal nome della divinità cui la festa era dedicata (Carmentalia, Quirinalia, Liberalia, etc.); ma si hanno anche casi più rari nei quali il nome della festa originava da quello della classe sacerdotale che operava in queste celebrazioni o del gruppo di cittadini cui era riservato quello specifico rituale (Lupercalia, Matronalia, Matralia). Un ulteriore gruppo di feste, invece, derivava il proprio nome dall'attività che si svolgeva in quella particolare giornata (Agonalia, Tubilustrium, Fordicidia, etc.) o da ciò che si intendeva commemorare (Regifugium, Parilia, Poplifugia, etc.); un ultimo gruppo, infine, è costituito dalle feste la cui denominazione prende origine dai luoghi in cui si celebravano questi rituali (Compitalia, Fontinalia, etc.). Però, più genericamente, possiamo affermare come vi sia un fil rouge che accomuna tutti i nomi delle antiche festività, ovvero la

39 Anche se, come già detto, è innegabile che il 19 marzo fosse anche una data del culto saliare: infatti Carisio (vd. nota 44) ricostruirà una para-etimologia del termine quinquatrus facendolo derivare da un ipotetico verbo quinquare, che però non è documentato nella lingua latina, il cui significato sarebbe quello di 'purificare', sottolineando così il carattere lustrale di questa giornata, derivatole dalle attività saliari.

40 Gli unici ad aver sottolineato l'unicità del termine Quinquatrus all'interno delle festività del ciclo numano sono la Invernizzi e quindi Rüpke: quest ultimo ha sostenuto, troppo frettolosamente, che "deren Etymologie nicht weniger kompliziert zu sein scheint als ihr kultischer Gehalt", facendo di questo sostantivo una 'eccezione' priva però di significato, dal momento che non avrebbe alcuna valenza religiosa e cultuale, a differenza degli altri nomi delle celebrazioni del feriale arcaico (RüPKE 1995, 264); la Invernizzi, invece, aveva già posto in debita evidenza il processo di sostantivazione dell'aggettivo quinquatrus, giustificando questo passaggio con delle celebrazioni legate al culto di Marte (INVERNIZZI 1994, 41). 
connotazione del divino, che si tratti del nome del dio, dei gruppi che vi prendevano parte o del luogo in cui esse si svolgevano; ma il sostantivo Quinquatrus, evidentemente, non ha nulla di 'divino', non rientra in alcuna delle categorie precedentemente elencate: non abbiamo un riferimento ad una divinità, né ad un qualsiasi rituale, bensì semplicemente una indicazione temporale, 'il quinto giorno', un riferimento oltretutto connotato da una particolare condizione di 'oscurità'. Ritengo, pertanto, che per comprendere la natura di questa festa arcaica, e quindi certificarne la pertinenza ad una specifica divinità, si debbano innanzitutto comprendere le motivazioni alla base di questa particolarità del nome; $\mathrm{o}$, in altri termini, cercare di individuare, attraverso il nome stesso, il culto ed il rituale ad esso sottesi.

\section{Il 'quinto giorno scuro'}

A questo punto, dunque, credo sia doveroso passare ad esaminare l'etimologia del nome, per risalire a quelle che sono state le origini della festa; un interesse linguisti$\mathrm{co}$, va sottolineato, che aveva coinvolto già nella tarda-repubblica gli eruditi romani: la prima fonte in merito è quella di Varrone, ${ }^{41}$ sulla cui linea interpretativa si porrà successivamente anche Festo; ${ }^{42}$ l'analisi varroniana si dimostra filologicamente corretta, in quanto l'erudito reatino risale all'origine del nome dal confronto linguistico con termini simili usati da altre popolazioni italiche, quali ad esempio i Falischi, in una sorta di comparativismo ante litteram: la desinenza -atrus, in questa analisi, è spiegata come un identificativo dei giorni successivi alle idi; ed in effetti, questo suffisso è da ricondurre all'espressione dies atri, che designerebbe comunemente tutti i giorni successivi alle idi, vale a dire i 'giorni oscuri'. Con il termine di 'giorno oscuro', pertanto, si intendevano tutti i giorni successivi al plenilunio, ovvero della seconda metà del mese, in quanto caratterizzati dalla luna calante e quindi da una diminuzione della luce durante la notte; il prefisso quinq-, invece, viene giustamente interpretato come derivante dal numero 'cinque', quinque. Una ulteriore prova della correttezza di questa esegesi, inoltre, risiede nel fatto che in vari calendari di altre popolazioni italiche, come dicevamo poc'anzi, sono documentati aggettivi similmente formati per designare i giorni dopo le idi, quali triatrus, sexatrus, septimatrus e decimatrus. ${ }^{43} \mathrm{~A}$ questo proposito, una interpretazione leggermente differente è quella che

41 Varr., Ling.Lat., VI, 14: Quinquatrus: hic dies unus ab nominis errore observatur, proinde ut sint quinque; dictus, ut ab Tusculanis post diem sextum Idus similiter vocatur sexatrus et post diem septimum septimatrus, sic hic, quod erat post diem quintum Idus, Quinquatrus.

42 Fest., 304L: Quinquatrus appellari quidam putant a numero dierum, qui fere his celebrantur. Quod scilicet errant tam hercule, quam qui triduo Saturnalia, et totidem diebus Compitalia; nam omnibus his singulis diebus fiunt sacra. Forma autem vocabuli eius, exemplo multorum populorum Italicorum enuntiata est, quod post diem quintum iduum est is dies festus, ut apud Tusculanos Triatrus, et Sexatrus, et Septematrus, et Faliscos Decimatrus. Minervae autem dicatum eum diem existimant, quod eo die aedis eius in Aventino consecrata est.

43 Torelli legge in questi aggettivi la testimonianza di feste similari, celebrate dalle altre popolazioni latine (ToRelli 1984, 198); in realtà questa interpretazione non si rende necessaria, al contrario appare piuttosto forzata, poiché queste espressioni, molto più semplicemente, devono essere considerate come ulteriori attestazioni dell'antico sistema di calcolo del tempo sulla base del ciclo lunare, che doveva essere 
ci tramanda Carisio, ${ }^{44}$ per il quale il termine -atrus della desinenza avrebbe indicato genericamente soltanto il giorno delle idi; ma in ogni caso, tutte e tre le fonti appena citate concordano comunque nell'attribuire alla prima parte della parola Quinquatrus, ovvero alla radice quinq-, il significato di 'quinto giorno'. Però, su questo tema, Carisio ci riporta anche una seconda interpretazione, sconosciuta agli altri autori, secondo la quale la radice quinq- deriverebbe da un ipotetico verbo quinquare, sinonimo di 'purificare, lustrare'; seppur erronea, dal momento che questo verbo non è attestato nella lingua latina, la para-etimologia del grammatico romano ci ribadisce il carattere purificatorio di questa giornata, in relazione alle cerimonie saliari. ${ }^{45}$ Aulo Gellio, infine, in un discorso di diversa natura sul significato della desinenza -triones all'interno della parola Septentriones, incidentalmente ci riferisce che, secondo il vulgus grammaticorum, se la radice quinq- indicava per l'appunto il numero di giorni che separavano questa data dalle idi, al contrario alla desinenza -atrus non si doveva attribuire alcun valore lessicale. ${ }^{46}$

Abbiamo, da ultimo, due ulteriori testimonianze, quelle di Ovidio e Porfirione, ${ }^{47}$ che vengono riportate semplicemente nella misura in cui danno conto dell'incertezza che regnava intorno al vero significato di questo nome in età imperiale, una esegesi che però viene incredibilmente accettata, ancora in tempi recentissimi, dal de Vaan: ${ }^{48}$ infatti, la radice quinq- viene spiegata da entrambi gli autori come il numero dei giorni nel corso dei quali si svolgeva al ciclo di feste in onore di Minerva; si tratta evidentemente di una paraetimologia, una errata interpretazione popolare sul nome del giorno, già radicata in epoca augustea, che sembra però avere avuto l'effetto pratico di allungare effettivamente il periodo festivo a cinque giorni, determinando la creazione di un unico lungo periodo di feste, terminante per l'appunto con il Tubilustrium e con un nuovo intervento dei Salii e degli ancilia..$^{49} \mathrm{Ma}$, al di là di esegesi erronee o para-etimologiche, la natura della parola Quinquatrus risulta chiara; la sua

comune ad altri popoli italici e del quale Varrone era sicuramente a conoscenza. Questi aggettivi, pertanto, dovevano soltanto indicare rispettivamente 'il terzo giorno dopo le idi', 'il quinto giorno dopo le idi', etc., e non altrettante festività.

${ }_{44}$ Char., Ars Gram., pag. 81, ed. Keil: Quinquatrus, sed non quinquatria. Non enim dictae sunt quinque dies sacrae, sed quod quintus dies Iduum, quas atrus antiqui dicebant; sive a quinquando, id est lustrando, quod eo die arma ancilia lustrari sint solita.

45 Nell'arco di questo giorno erano i Salii ad ancilia movere, atto di purificazione degli scudi sacri che successivamente venivano riportati nella Regia; a questa cerimonie lustrali era sicuramente collegata anche la danza dei Salii nel Comizio, alla presenza dei pontefici e dei tribuni Celerum (Fasti Praenestini ed Inscr.It. XIII, 2, 123). Warde Fowler sostiene che, seppur sia da considerarsi errata l'etimologia di Charisio, "but the reason given for it is valuable" (WARDE FowLER 1899, 58); Aust, invece, accetta questa esegesi (AUST 1899, 238 , n. 29).

46 Gell., II, 21, 7: vulgus grammaticorum 'septentriones' a solo numero stellarum dictum putat. 'Triones' enim per sese nihil significare aiunt, sed vocabuli esse supplementum; sicut eo, quod 'quinquatrus' dicamus, quod quinque ab Idibus dierum numerus sit, 'atrus' nihil.

47 Porph., II, 2, 197: Ac potius p. ut festis Quinquatribus olim. Quinquatres a quinque diebus, qui dies ad ferias Minervae pertinet, propterea, quod [est] festa sollemnitas eius deae per hoc spatium temporis celebratur. Ut igitur pueri ludo intenti insatiabiliter utuntur his feriis, ita actutum, inquit, moriturus exiguo vitae tempore raptim voluptatibus plurimis de[s]fruaris. Per il passo di Ovidio vd. nota 1.

48 DE VAAN 2008, 509.

49 Lyd., de Mens., IV, 60. 
etimologia è inoltre confermata dalla letteratura moderna che, con rare eccezioni in merito,${ }^{50}$ concorda sull'origine di questo termine: si trattava originariamente, infatti, di un comune aggettivo, genericamente usato per indicare il quinto giorno dopo le idi, di qualsiasi mese dell'anno, a partire dalla luna piena $;{ }^{51}$ come abbiamo già esposto, tutti i giorni successivi al plenilunio venivano definiti infatti come atri ed erano quindi contati progressivamente proprio a partire dal plenilunio, e non a ritroso come quelli della prima metà del mese. Questa prassi, è noto, era alla base del sistema romano di ricognizione del tempo fondato sull'osservazione del ciclo lunare: infatti, con le calende, "quando spuntava la luna nuova, si dava inizio al mese e si fissavano i termini della lunazione, cioè le nonae (il primo quarto) e le idus (plenilunio); in riferimento a questi due traguardi, il tempo era misurato dai giorni che occorrevano per raggiungerli, così che ogni giorno aveva un numero d'ordine sempre decrescente man mano che ci si avvicinava alle none e poi alle idi. Dopo le idi non esistevano più traguardi fissati in anticipo, dal momento che per l'inizio del mese-lunazione successivo si doveva aspettare che spuntasse la luna nuova: per questo inizialmente, dopo le idi, si contavano i giorni progressivamente proprio a partire dalle idi stesse." ${ }^{2}$

\section{Da 'quinto giorno scuro' a Quinquatrus}

Questo aggettivo, di conseguenza, era funzionale e quindi 'produttivo' da un punto di vista lessicale, soltanto in un sistema basato su di un calendario lunare, quindi sola-

50 Ribezzo, agli inizi del secolo scorso, proponeva il significato di 'festa dei cinque giorni', ipotesi non ricevibile, dal momento che considerava la parola come formata da quin(que)-quatrus, quindi con un valore di 'cinque ripetuto quattro volte', ma trovandosi poi costretto a giustificare le attestazioni di triatrus, sexatrus etc. come dei calchi dal nome Quinquatrus stesso (Ribezzo 1926, 263ss.). Hild sostiene, invece, che il termine atrus sarebbe una terminologia etrusca dei numeri ordinali (HILD 1969a, 802). La Porte, invence, nega inspiegabilmente l'evidente presenza della radice quinq-, ovvero 'cinque', nel nome della festa, non procurando però di dare una diversa spiegazione del termine (PORTE 1985, 122); successivamente però, in maniera altrettanto inspiegabie, accetta la teoria che la terminazione -atrus indichi i giorni dopo le idi (ibid., 242s.). Recentemente Humm è incorso in un grossolano errore, identificando le Quinquatrus con il Tubilustrium del giorno 23 -quando in realtà erano feste ben distinte, anche se collegate in un unico ciclo festivo- ("les Grandes Quinquatries correspondaient au jour du Tubilustrium") e quindi arrivando alla conclusione che tale data dovesse piuttosto corrispondere al quinto giorno prima delle calende di aprile; l'errata esegesi del termine è ancor più grave se si considera che, anche iniziando il computo a partire dal Tubilustrium, alle calende in realtà mancavano ancora ben 10 giorni e non solo 5! (Humm 2005, 472s.). De Vaan, da ultimo, è tornato ad affermare che la radice quinq- sia semplicemente da leggere come il numero dei giorni nel corso dei quali si svolgeva il ciclo di feste in onore di Minerva (DE VAAN 2008, 509), una interpretazione già proposta da Ovidio e Porfirione, ma evidentemente di natura paraetimologica (Ov., Fast., III, 809-810; Porph., II, 2, 197).

51 Warde Fowler 1899, 59; Wissowa 1902, 204, n. 2; WaCKernagel 1923-24, 215s.; Ernout Meillet 1932, 558; Walde - Hoffmann 1938, 408; Altheim 1951-53, I, 197s.; De Sanctis 1953, IV.2.1, 147; Hentschel 1963, 1150ss.; Kirsopp Michels 1967, 65, n. 16, 139; FraZer 1973, 144; SabbatucCi 1988, 131 s.; York 1986, 101s.; ArCella 1991, 27; RAdKe 1993, 141; RÜPKe 1995, 570ss.; Granino CeCere 2001, 33ss.; Donati - StefanetTi 2006, 36; LipKa 2009, 40. Dobbiamo però distinguere l'espressione di 'giorni scuri' in quanto successivi alle idi, dalla medesima definizione di dies atri data dallo stesso Varrone anche per designare i giorni seguenti alle calende, alle none ed alle idi (Varr., Ling.Lat., VI, 29), definizione che indicava i giorni nei quali non era possibile nihil novi incipere (sulle altre fonti a riguardo e più in generale sul significato di dies atri' cfr. KIRSOPP Michels 1967, 62ss.; PORTE 1985, 373ss.; RüPKE 1995, 570ss.).

52 Sabbatucci 1988, 132. 
mente in una fase notevolmente antica, quando per l'appunto l'arcaica comunità romana calcolava ancora lo scorrere del tempo sul moto e sulle fasi della luna; in effetti, una società che basasse il calcolo del tempo su di un sistema misto lunisolare già non necessitava più di una simile espressione, non aveva più bisogno di 'giorni dopo le idi', in quanto tutti i mesi dell'anno, e pertanto tutti i giorni, erano fissati in anticipo: infatti, già a partire dal calendario lunisolare, i giorni della seconda metà del mese si computeranno regressivamente, a partire dalle calende del mese successivo e non più progressivamente dal plenilunio del mese in corso. In altri termini, dunque, la presenza di un tale aggettivo ci riconduce ad un periodo decisamente alto, arcaico, della comunità romana, se consideriamo che un calendario prettamente lunare fu usato, al più tardi, solo fino alla metà del V sec. a.C., quando venne sostituito dall'introduzione del cosiddetto 'calendario pre-giuliano', probabilmente ad opera dei Decemviri. ${ }^{53}$ $\mathrm{Ma}$, a questo punto, credo sia necessario fare una ulteriore riflessione sul termine quinquatrus: abbiamo individuato, infatti, quale fosse il suo primigenio significato, ovvero una generica indicazione temporale in un calendario ancora esclusivamente lunare; inoltre, si è evidenziato come, nell'arcaico feriale romano, non vi fossero altre feste 'simili', etimologicamente parlando, ovvero que non esistessero altre celebrazioni il cui nome esplicitasse chiaramente un nesso con una qualsivoglia data o con un qualsiasi riferimento cronologico, aspetti che denotano inoltre una patente connotazione lunare della festività ${ }^{54}$ Pertanto, a mio avviso, dobbiamo interrogarci sulle ragioni di questa unicità del nome della festa: infatti, se un semplice aggettivo, funzionale originariamente alla misurazione del tempo, è assurto a nome proprio di una festa, è evidente che questa indicazione temporale fosse centrale, fondamentale, nei rituali che si praticavano in tale circostanza e quindi nel culto tributato alla dea; ritengo, infatti, che non vi sia altra spiegazione razionale ed accettabile che possa giustificare la sostantivazione di tale aggettivo, ovvero che possa rendere conto del passaggio da 'quinto giorno scuro' a Quinquatrus. E nel sottolineare l'importanza di questa evoluzione semantica dell'originario aggettivo quinquatrus, deve altresì essere evidenziato il fatto, altrettanto sostanziale e centrale nella nostra analisi, che la sua sostantivazione deve necessariamente essere avvenuta ed essersi fissata in seguito ad un lungo impiego come semplice riferimento temporale, ben prima della cessazione d'uso del calendario lunare alla metà del V sec. a.C.; ovvero, dobbiamo ritenere che

53 Cicerone (Rep., I, 16, 25) ci riferisce che nel 350esimo anno della città (circa il 400 a.C.) una eclissi solare si verificò alle nonae di giugno; ora, dal momento che una eclissi solare si può verificare solo con la luna nuova, è chiaro che alla fine del V - inizi del IV sec. il calendario non era già più in connessione con le fasi lunari. D'altro canto, da un passo di Ovidio si può forse evincere che furono i Decemviri a determinare la fine del calendario lunare in favore di uno lunisolare, dato che colloca la riforma calendariale alla metà del V sec. (Ov., Fast., II, 47-54). La vulgata moderna è concorde nell'attribuire ai Decemviri questo intervento (LATTE 1960, 36, n. 2; Kirsopp Michels 1967, 119ss.; RichaRd 1968, 58ss.; SAMUEL 1972, 166; MARTIN 1976, 239s.; York 1986, 21s.; BAYET 1992, 96; RÜPKe 1995, 204ss; Liou-Gille 2000, 42; HANNAH 2005, 106); anche per Guittard e Holleman, il periodo di utilizzo del calendario lunare va dall'arrivo dei re etruschi a Roma fino all'intervento dei decemviri del 450 a.C., e non è più in uso alla fine del V sec. a.C. (GUITTARD 1973, 213ss.; Holleman 1978, 202ss.), mentre per Flamant "le mois intercalaire du calendrier lunaire (ou peut-être luni-solaire) est attesté en 472; vers 450 les Décemvirs procèdent à une refonte de l'année” (FLAMANT 1984, 191ss.). (Per una storia degli studi anteriore ai lavori sopra citati, vd. KIRsOPP MicheLs 1967, 207ss.).

54 Sulla connotazione lunare delle Quinquatrus, e più in generale di Minerva, vd. CinAGLiA, e.p. 
la sostantivazione fosse già compiuta da lungo tempo al momento del passaggio al calendario pre-giuliano e che quindi le Quinquatrus fossero già nate per lo meno nel VI sec. ${ }^{55} \mathrm{Ma}$, d'altro canto, questa conclusione non può stupirci, dal momento che le Quinquatrus facevano parte delle festività del cosiddetto 'ciclo numano', ovvero del feriale arcaico, che avrebbe raggiunto la sua definitiva organizzazione, secondo la tesi oggi più accreditata, tra la fine del VII e gli inizi del VI sec. a.C. quale risultato di una lunga e complessa stratificazione. ${ }^{56}$

\section{Minerva e Anna Perenna}

Ma perché, dunque, il quinto giorno dopo le idi di marzo era talmente importante da determinare la sostantivazione del termine quinquatrus? Come precedentemente già affermato, questo processo deve essere necessariamente dipeso dalla centralità che $\mathrm{i}$ cinque giorni rivestivano nel culto e nel rituale; ${ }^{57} \mathrm{e}$ la sacralità del numero cinque in relazione a Minerva non è soltanto una ipotetica, seppur logica, deduzione: abbiamo infatti, incredibilmente trascurata dalla vulgata moderna, la testimonianza di Servio che afferma per l'appunto che hic numerus (scil. 'cinque') Minervae esse consecratus. ${ }^{58} \mathrm{Mi}$ sembra ovvio, di conseguenza, ritenere che la sacralità del numero 5 documentataci da Servio sia da correlare strettamente alla radice quinqu-all'interno del sostantivo Quinquatrus, ovvero ai ‘cinque giorni scuri' ed ai rituali ad essi connessi; in altre parole, dobbiamo ammettere che sia la sacralità del numero 5 , sia la sostantivazione dell'aggettivo quinquatrus, devono essere entrambe ricondotte alla centralità che sin dall'origine rivestiva il periodo di cinque giorni all'interno delle celebrazioni per Minerva. Ma se, dunque, la ragione della sacralità di questo periodo era tale da marcare il nome stesso della festa del 19 marzo, ritengo sia altresì fondamentale risa-

55 Per usare le parole di Guittard (1973, 204s.), "le cycle de quarantecinq jours de fêtes fixes, tel qu'il figure dans le calendrier préjulien, conçu pour prendre place à l'intérieur des divisions du mois en calendes, nones et ides, fondées sur les phases de la lune, ce cycle ne peut être que contemporain ou postérieur à l'introduction d'un mois lunaire à Rome".

56 Coarelli 1983, 186; ID. 2010, 338ss.; Pedroni 1998, 44s.; Carandini 2003, 419ss. Coarelli, seguito da Pedroni, propende invece per il periodo a cavallo tra VII e VI sec., agli inizi della monarchia 'etrusca', mentre Carandini ipotizza una relazione con l'opera di Servio Tullio e/o con la fondazione del culto di Giove Ottimo Massimo. Per Guittard, infine, la datazione più bassa ipotizzabile è la fine del VI sec. a.C., mentre la Liou-Gille propone una datazione alla fine del VI secolo a.C. con un influsso dell'astrologia dell'Asia Minore (GuitTard 1973, 204s.; Liou-Gille 1992, 317ss.).

57 Un paragone, mutatis mutandis, può essere prodotto con la festività cristiana della Pentecoste: le celebrazioni ed i riti del 'cinquantesimo giorno', infatti, sono evidentemente concepiti, e pertanto comprensibili, solo sulla relazione di questa festa con quella della Pasqua cristiana. Allo stesso modo, quindi, il 'quinto giorno dopo le idi' deve necessariamente sottendere una stretta relazione cultuale con la festa che si celebrava cinque giorni prima delle Quinquatrus, il 15 marzo.

58 Serv., in Georg., I, 277. L'unico studioso che menziona questa fonte è HiLd (1969a, 802 n. 3), il quale però attribuisce la sacralità del numero 5 all'ampliamento dei festeggiamenti per le Quinquatrus ad un periodo di cinque giorni, estensione databile presumibilmente in età medio-repubblicana; a mio avviso, questa interpretazione non è assolutamente accettabile: infatti, conosciamo numerose festività che si prolungavano per più giorni, da 3 a 5 sino a 7 giorni, ma non per questo i numeri or ora citati hanno mai acquisito alcun carattere sacro per le divinità titolari di quelle celebrazioni. 
lire all'inizio di questi cinque giorni, ovvero tornare al primo di essi per comprendere quale altra festività fosse all'origine delle Quinquatrus; dobbiamo, pertanto, esaminare la data e le celebrazioni del 15 marzo.

Alle idi, nel giorno del plenilunio, si celebravano i culti di Giove e di Anna Perenna; ma la connessione che stiamo cercando è evidentemente, ed esclusivamente, con quest'ultima divinità: infatti, come è stato già messo in luce da Torelli e Coarelli, ${ }^{59}$ nell'unico mito veramente romano cui prende parte Minerva, vediamo intervenire anche Anna Perenna, insieme a Marte; si tratta del famoso mito delle 'false nozze', noto grazie ai passi di Ovidio e Aulo Gellio: ${ }^{60}$ Marte, invaghitosi di Minerva, avrebbe interpellato Anna, nuper dea facta, per chiederle aiuto nel tentativo di conquistare la sua amata; ma la vecchia Anna, invece di lasciarsi convincere dal dio, se ne prende gioco apertamente sostituendosi a Minerva e presentandosi in vesti da sposa al momento delle nozze. Il mito, dal chiaro valore apotropaico e presente in numerose culture primitive, ${ }^{61}$ pone però soprattutto in evidenza la relazione calendariale, e quindi cultuale, tra le due dee, con Marte, dio titolare del mese in cui si celebravano le festività di Minerva ed Anna Perenna, coprotagonista in questa vicenda mitica. Il legame tra Anna e la nostra dea, suggerito dal termine Quinquatrus ed evidenziato anche dal mito, è altresì ribadito da un ulteriore dato, ossia dalla duplicazione delle Quinquatrus minusculae del 13 giugno: infatti, parallelamente a questa festa, istituita verosimilmente in epoca medio-repubblicana, viene duplicata anche la festa di Anna Perenna, il 18 giugno, come riportato dai Fasti Philocaliani, ${ }^{62}$ ovvero nel giorno immediatamente precedente quello del dies natalis del tempio di Minerva sull'Aventino. È evidente che la situazione di giugno rispecchi quella del mese di marzo e risulta inoltre altrettanto evidente, proprio dalle modalità della duplicazione, come nel ciclo delle festività di marzo la festa delle Quinquatrus fosse strettamente connessa con quella di Anna Perenna; evidentemente però il rituale di marzo non era più compreso con chiarezza quando, in epoca medio-repubblicana, si duplicarono questi culti: infatti, le Quinquatrus vennero collocate nel giorno delle idi, essendosi probabilmente perso l'originario significato della parola di 'quinto giorno scuro', mentre la nuova festa di Anna Perenna veniva invece celebrata in un giorno pari ed a ridosso del dies natalis della Minerva aventinese, e non più posta alle idi nel giorno della luna piena, come nel mese di marzo. ${ }^{63} \mathrm{Ma}$ la duplicazione esclusiva di queste due feste ribadisce e quindi certifica lo stretto nesso tra Minerva ed Anna Perenna, permettendoci di isolarle al contempo nel ciclo di feste saliari, all'interno del quale erano celebrate nel

59 Torelli 1984, 50ss.; CoARelli 1998, 209ss. Secondo l'interpretazione di Coarelli, la stessa epiclesi della dea, ovvero Capta, sarebbe da leggersi col significato di 'sposata', facendo della Minerva del Celio una divinità garante del matriomonio; una esegesi più credibile dell'epiteto della dea è però quella proposta dal filologo tedesco Köves-Zulauf che, 'rovesciando' il valore del verbo da passivo ad attivo, sulla base di numerosi altri esempi per divinità femminili di epoca arcaica (e.g. Mater Matuta, Iuno Februata, etc.), spiega il termine Capta col significato di 'colei che accoglie' (Köves-Zulauf 1993, 164s.). Dello stesso avviso anche FERRI (2006, 231ss.); per una storia degli studi e delle interpretazioni sul tema, vd. CinaGlia 2016.

60 Ov., Fast., III, 675-696; Gell., XIII, 23.

61 Questo tipo di rituale serviva ad allontanare il male dalla nubenda ed è tuttora presente anche in alcune società moderne, evidenziato da studi antropologici (FRAZER 1973, 125ss.; BoELS-JANNSEN 1993, 35).

62 Annae sacrum (Degrassi, Inscr. It., XIII 2, 249; Salzman 1990, 124).

63 Sulla duplicazione delle Quinquatrus minusculae vd. supra ed inoltre TorelLi 1984, 118. 
mese di marzo. Pertanto, possiamo con ragionevole certezza affermare che il culto di Minerva fosse collegato a quello di Anna Perenna, come evidenziato sia calendarialmente sia per quanto concerne il mito; ${ }^{64} \mathrm{ma}$, in altri termini, questo nesso tra le due dee altro non è se non una ulteriore conferma alla nostra originaria ipotesi, fondata inizialmente solo sulla base di un esame lessicale del sostantivo Quinquatrus, un termine che ci lasciava prefigurare delle celebrazioni strettamente connesse per quanto riguarda le date del 15 e del 19 marzo, ovvero all'inizio ed alla fine del periodo dei 'cinque giorni scuri'. Ma la 'sinergia' tra Minerva ed Anna Perenna non rimane solo in via ipotetica, esclusivamente a livello mitologico e calendariale; in realtà, le connessioni che si sono evidenziate sono più semplicemente il riflesso, la cartina al tornasole, di quello che era il ben più importante legame religioso che accomunava le due divinità: infatti, come è stato ampiamente dimostrato da Torelli, il nesso alla base di questa compartecipazione al medesimo ciclo cultuale è quello che vede entrambe le dee attive nei rituali di transizione femminile, a sovrintendere sulla comparsa del menarca e quindi sulla fase dei purgamenta virginalia, con le celebrazioni del 15 e 19 marzo che, oltretutto, inquadrano quella dei Liberalia, del 17, il giorno adibito all'acquisizione della toga virilis da parte dei pueri, venendo così a formare, all'inizio del nuovo anno, un unico periodo dedicato all'ingresso dei nuovi cives all'interno del corpo cittadino. ${ }^{65}$

\section{Conclusioni}

Una volta individuato l'innegabile collegamento tra il 15 ed il 19 di marzo anche dal punto di visto religioso, credo sia quindi comprovata anche la lettura e l'esegesi del termine Quinquatrus, ovvero come di un esplicito riferimento temporale alle idi ed al culto di Anna Perenna: il sostantivo Quinquatrus acquisisce valore, e la sua origine giustificazione, soltanto da questa relazione rituale e cultuale, tra le cerimonie che si celebravano alle idi del mese e quelle che si tenevano cinque giorni dopo, essendo le due celebrazioni accomunate in un unico periodo di purificazioni, un 'quindiale' sacro necessario al passaggio di status delle ragazze romane. Ma, come dimostrato in precedenza, dal momento che la sostantivazione dell'aggettivo quinquatrus si deve collocare al più tardi alla fine del VI sec. a.C., dobbiamo inoltre anche ammettere che questo nesso Anna Perenna - Minerva debba risalire ad un periodo notevolmente alto della religione romana; ovvero, che già in età arcaica il 'quinto giorno scuro' rappresentasse la fine di un periodo di cerimonie finalizzate al passaggio di status degli iuvenes romani. Di conseguenza, dobbiamo altresì concordare che la presenza della nostra dea nelle celebrazioni delle Quinquatrus sia altrettanto risalente; o meglio, che

64 Una ulteriore testimonianza della relazione tra Minerva ed Anna Perenna può verosimilmente essere considerata il ritrovamento, tra gli ex-voto del Santuario Orientale di Lavinium dedicato a Minerva, di una coppa a vernice nera databile al III secolo a.C. e sul cui fondo è inciso il nome ANA (Enea nel Lazio, 206, fig. D110; TORELLI 1984, 65).

65 Torelli 1984, 68ss.; Massa-Pairault 1987, 206ss.; Menichetti 1995, 84ss. Miller 2002, 217 ss. Carandini 2003, 562s.; Magini 2003, 76ss.; Moret 2012, 92 s. 
il culto di Minerva celebrato il 19 marzo sia all'origine della festa stessa, poiché era la nostra dea che, certificando la nuova condizione di nubendae per le ragazze romane, sanciva la fine del periodo di iniziazione. Ritengo, in conclusione, che non solo si debba riconoscere l'antichità del rapporto Quinquatrus - Minerva, ma soprattutto che la titolarità della festa sia da attribuire definitivamente, ed in toto, alla dea.

\section{Bibliografia}

Altheim, F. (1951-53): Römische religionsgeschichte, I-II, Baden-Baden.

ArCella, L. (1991): Fasti. Il lavoro e la festa. Note al calendario romano arcaico, Roma.

Aust, A. (1889): De aedibus sacris Populi Romani inde a primis Liberae Rei Publicae temporibus usque ad Augusti Imperatoris aetatem Romae conditis, Marburg.

Aust, E. (1899): Die Religion der Römer, Münster.

BAYET, J. (1992): La religione romana: storia politica e psicologica, Torino.

Blaive, F. (2003): "Du «Regifugium» aux «Equirria»: remarques sur les rituels romains de fin d'année", [in] C. Deroux - J. Dumortier-Bibauw (eds.), Hommages à Carl Deroux, 4. Archéologie et histoire de l'art (=Latomus 277), Bruxelles, 283-290.

BernsteIn, F. (1999): “Die römischen Ecurria/Equirria: kriegerische Feste?", Nikephoros 12, 149-169.

Boels-JANNSEN, N. (1993): La vie religieuse des matrones dans la Rome archaique, (=Collection de l'École française de Rome 176), Rome.

BöMER, F. (1957-58): P. Ovidius Naso. Die Fasten, Heidelberg, 2 vols.

Borgna, E. (1993): “Ancile e arma ancilia. Osservazioni sullo scudo dei Salii”, Ostraka II/1, 9-42

Brelich, A. (1949): Vesta (=Albae Vigiliae VII), Zürich.

Bremmer, J. N. (1993): "Three Roman aetiological myths", [in] F. Graf (ed.), Mythos in mythenloser Gesellschaft. Das Paradigma Roms, Stuttgart, 158-174 (http://dx.doi. org/10.1515/9783110974812.158).

Buchet, E. (2010): "La Grève des Tibicines", Bulletin de l'Association Guillaume Budé I, 174-196 (http://dx.doi.org/10.3406/bude.2010.2360).

Canciani, F. (1984): "Minerva", LIMC II.1, Zurich, 1074-1109.

CARANDINI, A. (2003): La nascita di Roma: dèi, Lari, eroi e uomini all'alba di una civiltà, II (=Biblioteca Einaudi 155), Torino.

Cinaglia, $\mathrm{T}$.

(2016): “Minervium vs. Minerva Capta: due facce della stessa medaglia?", 'Ilu. Revista de Ciencias de las Religiones 21, c.d.s.

(e.p.): "Minerva et Diana, quas ais pariter colendas: ovvero, la connotazione lunare di Minerva", Mythos.

CoARelli, F.

(1983): Foro Romano. I. Periodo arcaico, Roma. 
(1985): Foro Romano. II. Periodo repubblicano e augusteo, Roma.

(1996): “Minerva Capta, delubra; Minervium", LTUR III, Roma, 255.

(1997): Il Campo Marzio. Dalle origini alla fine della Repubblica, Roma.

(1997-98): "Il tempio di Minerva Capta e la domus di Claudio sul Celio", RendPontAcc 70, 209-218.

(2010): "Fasti Numani. Il calendario dei Tarquinii", AnnFaina 17, 337-353.

Degrassi, A. (1963): Inscriptiones Italiae. Vol. XIII - Fasti et Elogia. Fasciculus II - Fasti anni numani et iuliani, Roma.

De Sanctis, G. (1953): Storia dei Romani, Firenze, 4 vols.

Donati, N. - Stefanetti, P. (2006): Dies Natalis. I calendari romani e gli anniversari dei culti, Roma.

DuMÉZIL, G.

(1966): La religion romaine archaïque, Paris.

(1973): Mythe et épopée III. Histoires romaines, Paris.

Dury-Moyaers, G. (1981): Énée et Lavinium. À propos des découvertes archéologiques récentes (=Latomus 174), Bruxelles.

Enea nel Lazio (1981): AA. VV., Enea nel Lazio. Archeologia e mito: bimillenario virgiliano. Catalogo della mostra. Roma 22 settembre - 31 dicembre 1981, Campidoglio, Palazzo dei Conservatori [Mostra], Roma.

Ernout, A. - Meillet, A. (1932): Dictionnaire étymologique de la langue latine. Histoire des mots, Paris.

Flamant, J. (1984): “L’année lunaire aux origines du calendrier pré-julien”, MEFRA 96/1, 175-193 (http://dx.doi.org/10.3406/mefr.1984.1406).

FERri, G. (2006): “L'evocatio romana. I problemi”, StMatStorRel 30, 205-244.

Frazer, J. G. (ED. \& Trans.), (1973): Fastorum libri sex. The Fasti of Ovid. Volume 3: Commentary on Books III and IV, New York.

Gilbert, O. (1883-1890): Geschichte und Topographie der Stadt Rom im Altertum, Leipzig, 3 vols.

GIRARD, J. L.

(1970) "Les origines du culte de Minerve", REL 48, 469-472.

(1981): "La place de Minerve dans la religion romaine au temps du principat", $A N R W$ II.17.1, Berlin, 202-232.

(1989): "Minerva Capta: entre Rome et Faleries", REL 67, 163-169.

Graf, F. (2001): “Athena and Minerva. Two faces of one Goddess?”, [in] A. Deacy - A. Villing (eds.), Athena in the classical world, Leiden, 127-139.

Granino Cecere, M. G.

(2001): "Quinquatrus: tradizione popolare e tradizione antiquaria di una festività del calendario romano", ŽivaAnt 51, 25-38.

(2003): "Tibicines Romanorum qui sacris publicis praesto sunt tra Roma e Tibur", [in] M. L. Lazzarini - P. Lombardi (eds.), L'Italia centro meridionale tra Repubblica e Primo Impero. Alcuni aspetti culturali e istituzionali (=Opuscula Epigraphica 11), Roma, 75-91. (2014): "I Salii: tra epigrafia e topografia", [in] G. Urso (ed.), Sacerdos. Figure del sacro nella società romana. Atti del convegno internazionale Cividale del Friuli, 26-28 settembre 2012 (=I convegni della Fondazione Niccolò Canusio 12), Pisa, 105-128. 
GuitTARD, C. (1973): "Le calendrier romain des origines au milieu du Ve siècle avant J.-C.", $B A G B$ 2, 203-219 (http://dx.doi.org/10.3406/bude.1973.3229).

Hannah, R. (2005): Greek and Roman Calendars. Constructions of Time in the Classical World, London.

Hentschel, O. (1963): “Quinquatrus”, RE XXIV, Stuttgart, 1149-1162.

HILD, J. A.

(1969a): “Quinquatrus", DAGR IV.1, Graz, 802-804.

(1969b): “Salii”, DAGR IV.2, Graz, 1014-1022.

Hinds, S. (1992): “Arma in Ovid's Fasti. Part 1: genre and mannerism”, Arethusa 25/1, 81112.

Holleman, A. W. J. (1978): “Les calendriers préjuliens à Rome”, AntCl 47/1, 201-206.

Humm, M. (2005): Appius Claudius Caecus. La République accomplie (=Collection des Écoles françaises d'Athènes et de Rome 322), Rome.

InVERNIZZI, A. (1994): Il calendario (=Vita e costumi dei Romani antichi 16), Roma.

Jordan, H. - Huelsen, C. (1970-71): Topographie der Stadt Rom im Alterthum, Roma, 2 vols.

Jullian, C. (1969): “Flamen, Flaminica, Flamonium”, DAGR II.2, Graz, 1156-1188.

Kirsopp Michels, A. (1967): The Calendar of the Roman Republic, Princeton.

Köves-Zulauf, T. (1993): “Minerva Capta - Eine gefangene Göttin?”, [in] J. Dalfen - G. Petersmann - F. F. Schwarz (eds.), Religio Graeco-Romana. Festschrift für Walter Pötscher (=Grazer Beiträge Suppl. 5), Horn, 159-176.

La BuA, G. (2010): “Minerva Capta (Ovidio Fasti 3, 809-848)”, [in] G. La Bua (ed.), Vates operose dierum: studi sui Fasti di Ovidio (=Testi e studi di cultura classica 48), Pisa, 51-63.

LATTE, K. (1960): Römische Religionsgeschichte, München.

Liou-Gille, B. (1992): “Le calendrier Romain: histoire et fonctions (Tite Live, I 19, 6-7)", Euphrosyne 20, 311-322.

(2000): “Les Agonia, le «rex sacrorum» et l'organisation du calendrier", Euphrosyne 28, 41-60.

LIPKA, M. (2009): Roman gods. A conceptual approach, Leiden-Boston.

LoICQ, J. (1964): “Le témoignage de Varron sur les Ecurria”, Latomus 23, 491-501.

Magdelain, A. (1990): “Quando rex comitiavit fas", [in] A. Magdelain - Y. Thomas (eds.), Ius imperium auctoritas. Études de droit romain (=Collection de l'École française de Rome 133), Rome, 271-277.

Magini, L. (2003): Astronomia etrusco-romana, Roma.

MAIURI, A. (2009): "L'equipaggiamento saliare tra funzionalità estetica e simbologia sacrale", [in] S. Botta (ed.), Abiti, corpi, identità. Significati e valenze profonde del vestire (=Alti studi di storia delle religioni 8), Firenze, 149-168.

Marcattili, F. (2011): "Sacris in postibus arma (Verg., Aen., 7, 183). Guerra, Lemures e liturgie romane del ritorno", [in] D. Loscalzo - C. Masseria (eds.), Miti di guerra, riti di pace. La guerra e la pace. Un confronto interdisciplinare, Bari, 251-258. 
MARTin, P. M. (1976): "La fonction calendaire du roi de Rome et sa participation à certaines fêtes", Annales de Bretagne et des pays de l'Ouest 83/2, 239-244 (http://dx.doi. org/10.3406/abpo.1976.2808).

Martínez-Pinna, J. (1980): "La danza de los Salios, rito de integración en la curia", AEspA 53/141-142, 15-20. (2012): "Los ludi en la Roma arcaica", De Rebus Antiquis 2/2, 152-179.

Massa-Pairault, F. H. (1987): "De Préneste à Volsinii: Minerve, le fatum et la constitution de la société", ParPass 42, 200-235.

Menichetti, M. (1995): ... Quoius forma virtutei parisuma fuit ... Ciste prenestine e cultura di Roma medio-repubblicana, Roma.

Miller J. F. (2002): “Ovid's Liberalia”, [in] G. Herbert-Brown (ed.), Ovid's Fasti. Historical Readings at its Bimillennium, Oxford, 199-224 (http://dx.doi.org/10.1093/acprof:oso/9780198154754.003.0009).

Moret, J.-M. (2012): “Le feste dei nani”, [in] C. Bocherens (ed.), Nani in festa. Iconografia, religione e politica a Ostia durante il secondo triumvirato (=Bibliotheca Archaeologica 26), Bari, 49-108.

Pedroni, L. (1998): “Ipotesi sull'evoluzione del calendario arcaico di Roma”, PBSR 66, 3955.

Penny Small, J. (1982): Cacus and Marsyas in Etrusco-Roman Legend, Princeton.

Phillips, C. R. (2006): “Minerva. Cult”, [in] H. Cancik - H. Schneider (eds.), Brill's Encyclopaedia of the Ancient World, VIII, Leiden - Boston, 939-943.

Platner, S. B. - Ashby, T. (1965): A Topographical Dictionary of Ancient Rome, Roma (http://dx.doi.org/10.1017/CBO9781316219706).

Porte, D. (1985): L'etiologie religieuse dans les 'Fastes'd'Ovide, Paris.

RADKe, G. (1993): "Römische Feste im Monat März”, Tyche 8, 129-142.

REINACH, A. (1969b): “Tibia”, DAGR V, Graz, 300-342.

(1969a): “Tuba”, DAGR V, Graz, 522-528.

Ribezzo, F. (1926): “Quinquatrus”, RIGI 10/1, 100 e 263-265.

RichARD, C. (1968): “Le calendrier préjulien”, REL 46, 54-62.

Richardson, L. (1992): A New Topographical Dictionary of Ancient Rome, Baltimore.

Ross TAYLOR, L. (1923): Local cults in Etruria (=Papers and Monographs of the American Academy in Rome 2), 1923.

RüPKE, J. (1995): Kalender und Öffentlichkeit, Die Geschichte der Repräsentation und religiösen Qualifikation von Zeit in Rom (=Religionsgeschichtliche Versuche und Vorarbeiten 40), Berlin-New York.

SABBATUCCI, D. (1988): La religione di Roma antica: dal calendario festivo all'ordine cosmico (=La Cultura 67), Milano.

Salzman, M. R. (1990): On Roman Time: the Codex-calendar of 354 and the Rhythms of Urban Life in Late Antiquity (=Transformation of the Classical Heritage 17), Oxford.

Samuel, A. E. (1972): Greek and Roman Chronology. Calendars and Years in Classical Antiquity, München. 
SCHEID, J. (2008): "Il culto di Minerva in epoca romana e il suo rapporto con la Minerva di Travo", [in] AA.VV., Minerva Medica in Valtrebbia. Scienze storiche e scienze naturali alleate per la scoperta del luogo di culto (=Quaderni di Archeologia dell'Emilia Romagna 19), Piacenza, 85-91.

Schilling, R. (1992): Ovide. Les Fastes, vol. I, Paris.

SChÜRmANN, W. (1985): Typologie und Bedeutung der stadtrömischen Minerva-Kultbilder (=Rivista di Archeologia suppl. 2), Rome.

Scullard, H. H. (1981): Festivals and Ceremonies of the Roman Republic, London.

Siebert, A. V. (2002): “Tubilustrium”, Neue Pauly 12/1, Stuttgart, 889.

Storchi Marino, A. (1979): “Artigiani e rituali religiosi nella Roma arcaica“, RendNap 54, 333-357.

TORELLI, M.

(1982): Typology and structure of Roman historical reliefs, Ann Arbor (http://dx.doi. org/10.3998/mpub.8062).

(1984): Lavinio e Roma. Riti iniziatici e matrimonio tra archeologia e storia, Roma.

(1990): "Riti di passaggio maschili di Roma arcaica", MEFRA 102, 93-106 (http://dx.doi. org/10.3406/mefr.1990.1661).

DE VAAn, M. (2008): Etymological Dictionary of Latin and the other Italic Languages, Leiden-Boston.

VENDRIES, C. (2004): "Musique romaine", ThesCRA 2, 397-415.

VetTER, W. (1936): “Tibia”, RE VI A 1, Stuttgart, 808-812.

WaCKernagel, J. (1923-24): “Dies ater”, ARW 22, Leipzig-Berlin, 215-216.

Walde, A. - Hoffmann, J. B. (1938): Lateinisches etymologisches Wörterbuch, I-II, Heidelberg.

Warde Fowler, W. (1899): Roman Festivals of the Period of the Republic. An Introduction to the Study of the Religion of the Romans, London.

Wissowa, G.

(1899): “Capta”, RE III.2, Stuttgart, 1554.

(1902): Religion und Kultus der Römer, München.

Woodard, R. D. (2002): "The disruption of time in myth and epic", Arethusa 35/1, 83-98 (http://dx.doi.org/10.1353/are.2002.0014).

York, M. (1986): The Roman Festival Calendar of Numa Pompilius (=American University Studies. Series XVII, Classical Languages and Literature 2), New York.

ZIolkowski, A. (1992): The temples of mid-republican Rome and their historical and topographical contest (=Saggi di Storia Antica 4), Roma. 